\title{
The Role of Speech Audiometry in Practical Otology
}

\author{
BY D. R. HAYNES, M.B., B.Ch. (Rand), D.L.O. (R.C.P. \& S.) \\ Registrar, Johannesburg General Hospital.
}

It is only recently that a method for measuring hearing in terms of speech discrimination has passed from the field of laboratory research to the practical field within easy reach of the practising otologist.

We may well ask how large a part machines are entitled to play in the practice of medicine and I have heard audiometry condemned by one of the eminent American otologists on the grounds that it does not make a diagnosis and it is not necessary in the treatment of ear disease. In both respects I think he is wrong, but his being wrong is not quite as obvious as his being right appears to be.

The majority of cases of Meniére's disease may be diagnosed on the history alone. Nobody, however, would think of making a diagnosis of the disease without carrying out a complete clinical examination of the patient, including tuning fork tests. Nobody should diagnose the condition without carrying out tests of labyrinthine function. And, when all this has been done, there will remain a small number of cases in which audiometry gives the final clue to diagnosis when the other tests have failed.

The course of disease is of tremendous importance in its treatment and in assessing the progress of hearing or of deterioration of hearing, audiometry plays perhaps an even greater part than it does in the diagnosis. Modern neuro-otology, as this branch of surgery is called by Cawthorne, would scarcely be justified if it were not for our ability to measure and record patients' hearing.

Speech audiometry by itself is not a complete measurement of hearing ability and in the first place pure-tone audiometry should be employed. There are a great many advantages to the use of speech audiometry but unfortunately it cannot provide all the answers usually required in assessing hearing.

The disadvantages of speech audiometry are for the most part of a technical nature. It is easy, for instance, to obtain a perfectly satisfactory pure-tone audiogram from an unintelligent subject. In fact, my experience has been that the unintelligent subject usually gives a more consistently reproducible audiogram than the intelligent, introspective type of person.
If standard, comparable results are to be obtained in speech audiometry, the subjects must be reasonably intelligent and, to a certain extent, educated. In this regard, I must mention the speech audiograms that were taken of a professor of languages at one of the American universities. His mother tongue was German but he taught in English and French and spoke all three languages fluently. In spite of this the speech audiograms in the three languages showed a very much better discrimination for the German word lists than for either of the other two.

Here was a highly educated, intelligent man whose audiograms for the extra languages might have been interpreted as showing $\alpha$ hearing loss when there was none in fact.

In exactly the same wory, differences in dialect may give results in speech audiometry for uneducated subjects which are not commensurate with the pure-tone audiograms.

To overcome these difficulties to a certain extent, the monitored live voice may be employed instead of calibrated records but this does not altogether obviate the undersirable features of the test.

It becomes obvious now that assessment of hearing must be done with pure-tone and speech audiograms side by side.

A good exercise is to forecast the speech audiogram after examining the amount of residual hearing for the accepted speech frequencies on the pure-tone audiogram. This can usually be done with a fair degree of accuracy in the case of conductive deafness. With many cases of perceptive deafness, however, the forecast is found to be grossly inaccurate and this applies particularly in those cases exhibiting the phenomenon of recruitment.

Here it is of interest to mention that an intelligent adult can obtain a high discrimination score even if he has no hearing for frequencies above 500 c.p.s. Using the master hearing aid such as that used for research in hearing aids by the British Medical Research Council, it is possible to cut out all frequencies above 500 c.p.s. and yet obtain good speech audiograms, even with p.b. word lists.

In practice, speech audiograms can be done quickly, especially when a rough assessment 
of hearing for speech is made according to the pure-tone audiogram. In cases of conductive deafness, the curve will be parallel to the normal curve. In perceptive deafness, the curve flattens out as the percentage of discrimination increases and it may never reach the $100 \%$ level. When the phenomenon of recruitment is present, the percentage discrimination actually falls with the increase of intensity above a certain level and it is therefore essential in cases of recruitment to complete the audiogram in order to get the whole picture.

\section{CONDUCTIVE DEAFNESS.}

From the patient's point of view, the most important aspect of hearing is his hearing for speech.

With conductive deafness, the speech audiogram follows a regular pattern and can be assessed with a fair degree of accuracy from the pure-tone audiogram.

Our experience with fenestration surgery has led us to believe that if we wish accurately to determine the improvement or otherwise of hearing following surgery we must have speech audiograms as well as pure tone audiograms.

The best illustration of this is a feature that I have noticed repeatedly with fenestrations, regardless of how expert the surgeon might be. In attempting to assess the suitability of cases for fenestration, the otologist usually regards the patient with a rising curve in the high frequencies on pure tone audiograms as having the better prospects of success than the patient with a straight curve or with $a$ curve dropping in the high frequencies.

Yet there is a very large proportion of fenestrated cases whose post-operative puretone audiograms show a very successful rise in the hearing over the speech frequencies but a marked drop in the high frequencies. In other words, the post-operative curve is reversed. If an aggregate of all frequency changes were to be made in these cases, the sum would probably be zero.

The speech audiograms give a completely different picture in these successful fenestrations and the curve is merely moved bodily to the left which is much more in keeping with the general clinical expectations.

This fact is then a very important one in making pre- and post-operative speech audiograms an essential part of fenestration surgery.

\section{PERCEPTTVE DEAFNESS.}

The attempt to match hearing aids to puretone audiograms has been a failure. This may be possible in cases of conductive deafness but with perceptive deafness trial and error becomes a necessity for fitting a hearing aid.

Once again, the speech audiogram is in itself a very much better indication of suitability for hearing aid than is the pure-tone audiogram by itself. If recruitment is shown to be present on a speech audiogram, then it is at once obvious that pure amplification of sound over all frequencies will not benefit the patient. These are the cases in which there is an indication for 'modification of the hearing aid by means of automatic loudness control.

The speech audiometer in a case of this sort acts as a master hearing aid and if the speech audiogram shows a marked degree of recruitment it may be assumed that the patient will not benefit from the use of a hearing aid.

\section{RECRUTTMENT.}

Speech audiograms in themselves may give a very much better indication of the degree of recruitment present than the more tedious and difficult tests of loudness balance. They have the further advantage of indicating recruitment when both ears are affected, whereas the pure-tone audiograms are of use only when one ear is affected and the other ear normal.

If loudness balance tests are to be reliable, they must be carried out over several frequencies and the time necessary to do this is considerable. Furthermore, the patient is easily fatigued and usually two or three attempts are necessary to complete the test.

The only condition in which recruitment regularly occurs is Meniere's Disease and for this reason speech audiometry is an essential part of the investigation of this condition. In spite of the fact that most modern audiometers are fitted with loudness balance attachments, the only satisfactory wory of carrying out accurate tests for loudness balance is by the use of two separate, equally calibrated or corrected audiometers or their equivalent. The tests should be done for at least three frequencies and because of this the test is scarcely to be regarded as of great value to the practising otologist.

Speech audiometry is readily available nowadays to any otologist and speech audiograms are easily done so that here is a practical indication of recruitment that has a tremendous diagnostic value.

Occasionally, a mild degree of recruitment is present in cases of presbyacusis and it may, of course, occur immediately after trauma to 
the labyrinth. In the latter condition, the recruitment tends to disappear with the passage of time after the trauma.

\section{CHILDREN.}

Speech audiometry has a special place in the assessment of hearing in children. Puretone audiometry is notoriously inaccurate in children and it is extremely difficult to reproduce accurate audiograms.

Speech audiograms, however, may be very successfully employed in children of average intelligence over the age of four years.

If records are made with a carrier phrase, such as "You will now say...." there is very little difficulty in obtaining accurate audiograms which maintain the interest of the child. These audiograms are used in children up to the age of 10 or 12 years and in the occasional cases of Meniére's Disease encountered in this age group, the above remarks on the assessment of recruitment apply even more strongly.

\section{SOCIAL ADEQUACY INDEX.}

The Social Adequacy Index is designed to give an indication of the intelligibility of speech for a particular subject. It is a combined index of hearing ability and interpretation and the pure-tone audiogram plays no part in arriving at the index.

While there is no practical value to assessment of percentage of hearing, the Social Adequacy Index has a definite value and gives a truer reflection of "social hearing ability" than does a percentage figure based upon pure-tone audiometry.

Unfortunately, there are far too many factors which influence hearing and the S.A.I. takes into account only two of them, so that it cannot truly be regarded as an index of social adequacy.

The index can be determined if speech discrimination does not reach the $100 \%$ level even at the highest amplification. It cannot be determined, however, if recruitment is present and the percentage discrimination actually decreases with an increase in amplification.

\section{MALINGERING.}

Numerous tests for feigning deafness have been devised and naturally some tests are better than others, but the malingerer simulating deafness may be difficult to detect with certainty.

A new test is being corried out in Sweden in which the speech audiometer is used and it appears to be almost impossible to simulate deafness effectively when this test is employed.
The suspected malingerer is given an article to read and a tape recording of his speech is made. This recording is played back to the patient after a time lag of one third of a second. If he is in fact malingering, he finds it impossible to concentrate on what he is reading and begins to falter and stammer and becomes utterly confused within a few seconds.

\section{CONCLUSION.}

I have attempted to show that speech audiometry should be regarded as an essential part of modern otology

Only a few years ago, otology consisted for the most part of surgery of suppurative ear disease. In the last 15 years, great advances in otologic surgery have coincided with advances in the treatment of infections of all kinds.

Contrary to frequently expressed opinion, the relief from treatment of suppurative ear conditions has made possible a greater concentration on the surgical treatment of deafness and has thereby widened the scope of otology.

Lempert designed the modern fenestration operation and thereby brought practical hearing within the reach of millions of sufferers from otosclerosis. Hallpike described the pathology of Meniére's Disease and Cawthorne designed his operation for labyrinthectomy which can be regarded as a cure for unilateral Meniére's Disease.

There is a considerable emphasis at the present time on the plastic surgery of the tympanic membrane and it is to be hoped that these procedures will be productive of more useful hearing in the future.

We have to await the results of the operation for mobilization of the stapes in otosclerosis and we may be on the brink of a further big advance here.

Because of the advances in otologic surgery, new standards have been set in all the auxiliary services and we have now reached the stage where speech audiometry is an essential auxiliary service.

I do not suggest that because we have speech audiometry, that the older methods of investigation and examination may be discarded. On the contrary, I emphasise that complete examination of hearing now consists of clinical examination, including tuning fork tests, pure-tone and speech audiograms.

It is only by accepting this as a new standard in practical otology that we can hope to achieve the best results from management of the deaf patient, ALL THE TIME. 